\title{
An efficient machine learning-based COVID-19 identification utilizing chest $\mathrm{X}$-ray images
}

\author{
Mahmoud Masadeh ${ }^{1}$, Ayah Masadeh¹, Omar Alshorman², Falak H Khasawneh ${ }^{3}$, Mahmoud Ali \\ Masadeh $^{4}$ \\ ${ }^{1}$ Computer Engineering Department, Yarmouk University, Irbid, Jordan \\ ${ }^{2}$ Faculty of Engineering and AlShrouk Trading Company, Najran University, Najran, Saudi Arabia \\ ${ }^{3}$ Department of Applied Medical Sciences, Zarqa University College, Al-Balqa Applied University, Al-Salt, Jordan \\ ${ }^{4}$ Ministry of Education, Amman-Al Abdli, Amman, Jordan
}

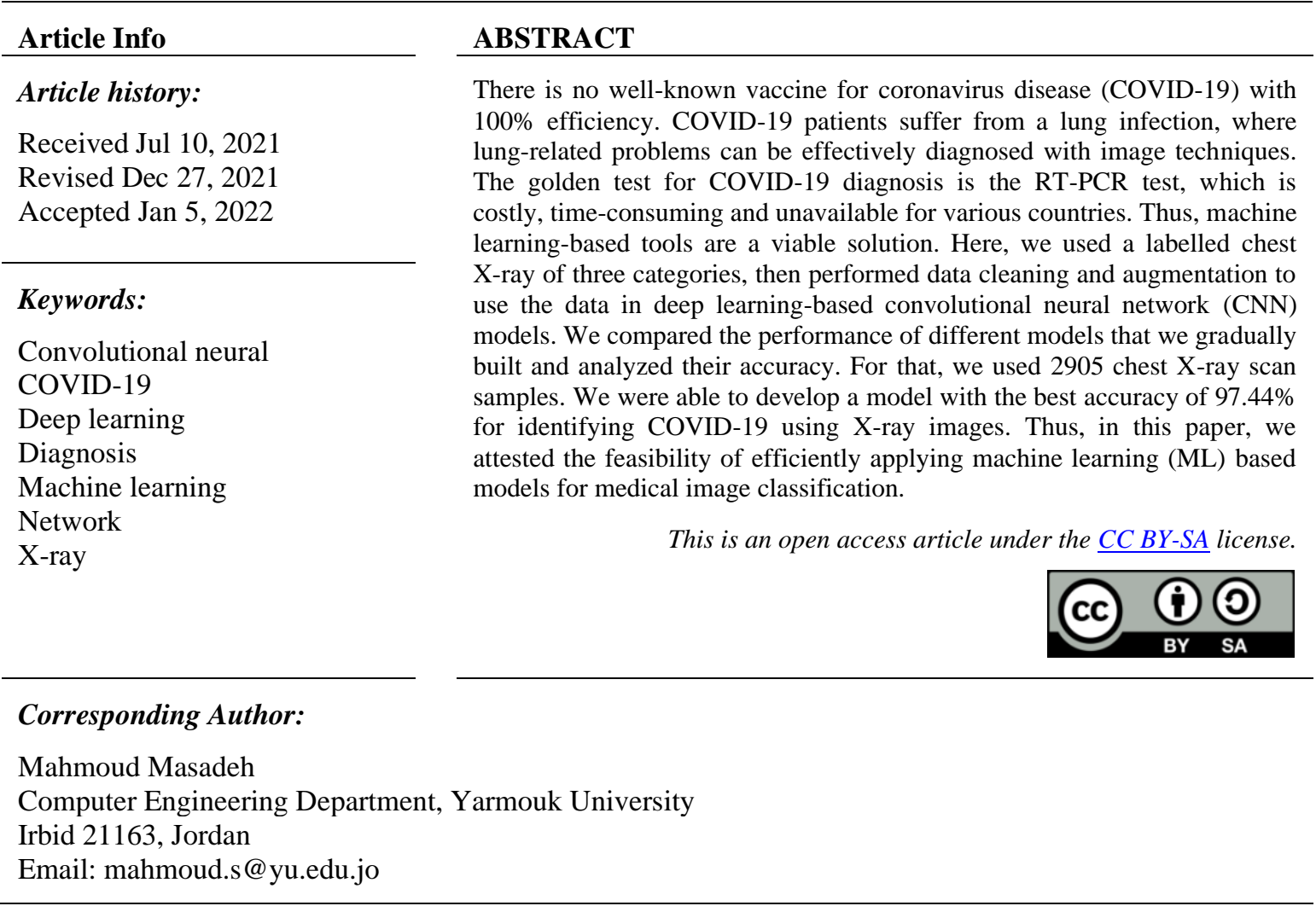

\section{INTRODUCTION}

Currently, the entire world is suffering due to the coronavirus disease (COVID-19). Most of the countries all over the world started vaccinating their people for COVID-19 [1]. Thus, vaccines generate protection against the illness, as a consequence of developing an immune response to the SARS-Cov-2 virus. However, COVID-19 is still threatening global health care, since none of the widely used vaccines has approved $100 \%$ efficiency without any side effect [2]. Moreover, the ability of the countries to obtain the vaccine is as low as their ability to afford reverse transcription-polymerase chain reaction (RT-PCR) tests for their residents [3]. As of 26 Dec 2021, the total number of cases confirmed to be infected has surpassed 238 million, in more than 220 countries, with more than 4.82 million deaths from COVID-19. Thus, it has caused calamitous consequences on everyday activities, health care, and global economics. Figure 1(a) shows the average number of worldwide new cases per week while Figure 1(b) shows the average number of worldwide deaths per week. As shown in Figure 2 the number of people, as 25 Sept 2021, who have received at least one dose of a vaccine is $44.8 \%$ of the world population, while the number of people who fully vaccinated is around $32.9 \%$ of the world population. However, more than 20 countries did not reach $1 \%$ yet while 40 countries have less than $5 \%$ of the population being vaccinated. Such numbers show that reaching to 
$100 \%$ vaccinated world is a long-journey, specially for low-income countries, where the best solution is COVID-19 avoidance and early detection [4].

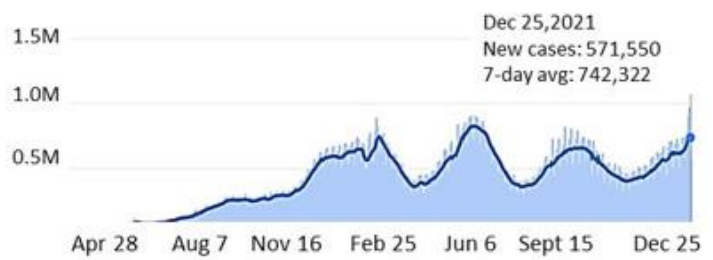

(a)

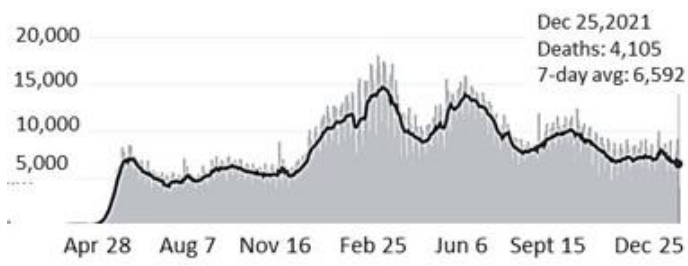

(b)

Figure 1. Average number of world wide (a) new cases per week and (b) deaths per week

Since the beginning of the pandemic, different repositories, e.g., Github and Kaggle, presented online chest X-ray images for natural and infected peoples where such images include notable knowledge regarding the COVID-19 virus [5]. The diagnosing process of COVID-19 required the direct contact of the infected patients with the medical staff, which is very risky task [6]. Thus, more precautions are required. Moreover, the treatment procedure should be strict to reduce the risk of infecting the healthy ones [7]. This plague is developing in a sequential process that conveys from one person to another after contacting COVID-19 infected bodies [8]. The diagnosis process of this epidemic includes various personnel, e.g., doctors, nurses, lab technicians and hospital staff [9]. Therefore, to degrade the effect of COVID-19 several approaches have been applied [10]. Moreover, medical imaging is an effective methodology for examining and forecasting the influence of COVID-19 on an individual's well-being [11].

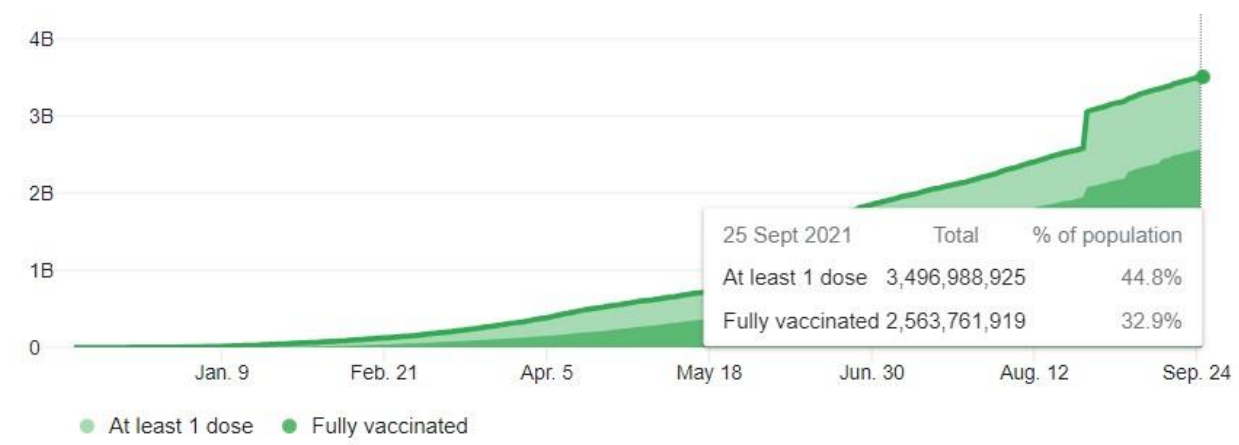

Figure 2. The number of people worldwide who have received a minimum of one dose of a vaccine

Medical systems, including the healthcare givers, are facing tremendous demands on the available resources, e.g., medical tools, RT-PCR test kits, and hospital beds [12]. Thus, the ability to offer a prioritized hospitalization for severe cases is crucial, where the inability to do so exacerbates the plague rate and significantly hinders disease containment [13]. To help the medical systems, especially in the developed countries with limited resources, various prediction models have been introduced for fast, cheap, and reliable diagnosis of COVID-19 [14].

The main symptoms for COVID-19 include respiratory dysfunction with moderate to critical conditions, e.g., sore throat, coughing, and diarrhea, where the virus circulates by air and physical contact [15]. Moreover, elderly people with chronic diseases need special handling since they are vulnerable and 
could exhibit serious breakdown if affected with this disease. Thus, the most familiar response to this outbreak is patient isolation and infection-control standards. Whenever the RT-PCR test kits are unavailable due to time and/or cost constraints, machine learning-based approaches could be used, where the smart analysis of X-ray and computerized tomography (CT) images can easily identify COVID-19 cases [16]. However, there is no golden ML-based model where a highly accurate approximation is acceptable in such cases [17], [18].

Various ML-based techniques are used for disease detection using medical images and signals, e.g., electroencephalogram (EEG) [19], electrocardiogram (ECG), and electromyography (EMG) [20]. For example, the authors of [21] introduced a ML-based decision assistance scheme for the prediction of diabetes, while the authors of [22] used various ML-based techniques for cancer detection. The performance of such ML-based approaches is extremely influenced by the quality of the non-optimal and time-consuming hand-engineered features [23]. Therefore, implementing an automatic extraction of optimal features, from the input data, for efficient feature extraction with enhanced classification precision, is required. Thus, various techniques based on deep learning (DL) could be utilized [24], [25].

The authors of [26] suggested using deep CNNs for segmenting lung X-ray images. Thus, they were able to enhance the performance of clinical diagnosis of different diseases of the lungs. In [27], the authors applied CNNs for automatic feature learning and classification. Then, revealed a reliable system to recognize breast cancer using histology images [28]. However, using DL models requires large datasets for training, where such a huge dataset is not always available. Moreover, the majority of medical images datasets are imbalanced with inadequate representation, in addition to privacy and confidentiality concerns of medical data [29]. Therefore, different data augmentation methods are introduced to defeat the shortage of labelled data and model over-fitting. For that, data alteration, e.g., scaling, flipping, rotation, and transformation, is used [30].

According to [31], there exist three main mechanisms to utilize CCNs for the classification of medical images; preparing the CCN from whatever is available (the scratch); using already pre-trained (offthe-shelf) CCNs; unsupervised CCN pre-training with supervised fine-tuning. In this work, we used the first mechanism (trained the $\mathrm{CCN}$ from the scratch). In this paper, we introduce an efficient DL-based model that predicts a positive SARS-CoV-2 infection based on X-ray images. We utilized a well-known machine learning method, $\mathrm{CNN}$, which is effective to realize. The proposed model is also able to recognize pneumonia as well as normal cases. We trained the model on publicly available data, i.e, Kaggle repository, which is used by various related works. Thus, our model can be realized globally for efficient monitoring and prioritization of testing for the virus in the worldwide community.

Next, we describe some of the work that is closely related to this paper based on utilizing X-ray and CT images for disease diagnosis. The authors of [32] suggested a Multi-level thresholding and SVM based methodology for COVID-19 detection utilizing 40 images of chest X-ray where the obtained accuracy was 97.48\%. Similarly, the authors of [33] introduced a novel framework of DL to diagnose COVID-19, called COVIDX-Net. Based on 50 images of chest X-ray, it supports the practitioners to automatically detect COVID-19. Based on 25 COVID-19 positives and 25 normal images, the accomplished accuracy was $90 \%$.

The scholars of [34] introduced various approaches for COVID-19 discovery with the challenges facing the RT-PCR. Thus, it is highly obliged to realize an automatic identification method to restrict the expanse of the virus through direct communication. While there are various DL approaches for COVID-19 detection, they are able to detect subjects suffering from pneumonia without the ability to accurately specify the exact cause of pneumonia, i.e., whether it is COVID-19, bacterial, or fungal attack. The authors of [35] proposed using AI tools, by radiologists and healthcare professionals, for fast and reliable COVID-19 diagnosis. Due to a shortage of publicly available datasets, the authors built a dataset of $170 \mathrm{X}$-ray images from multiple sources. Then, build a forecasting technique based on DL and transfer learning algorithms. Experimental results reveal a $94.1 \%$ accuracy based on modified CNN.

In [36], the authors designed a DL-based model, i.e., COVID-Net, which delivered a $92.4 \%$ success rate using 13975 radiography images. The used images are collected from various open access data. A large dataset of $1427 \mathrm{X}$-ray images was collected in [37], where the outcomes indicate that DL with X-ray imaging can obtain meaningful biomarkers related to the COVID-19 disease with an accuracy of 96.46\%. In their study, the authors of [38] investigated the realization of various classification models for COVID-19 detection where they practiced eleven different $\mathrm{CNN}$ models. The best classification model realized accuracy of $95.33 \%$ for the identification of COVID-19, where that model was based on a deep feature plus SVM. The study of [39] proposed five pre-trained CNN-based models for COVID-19 detection using chest X-ray images. The models InceptionV3 and ResNet50 achieved an average accuracy of $95.4 \%$ and $96.1 \%$, respectively.

The authors of [40] trained a DL-based model on the ResNet-101 CNN architecture using a dataset of 4376 X-ray images. They achieved an accuracy of $71.9 \%$, where such low accuracy is due to the shortage 
of the used images in the testing stage. Various work proposed using CT images for COVID-19 diagnosis, e.g., [41]-[44], which achieved an accuracy of $86 \%, 82.9 \%, 90.8 \%$, and $86.7 \%$, respectively. It is known that a chest X-ray is a reliable first-look exam with low cost, while a CT scan is suitable for exact analysis and therapy with high cost. Moreover, CT radiation causes attention for subjects who need dynamic monitoring, in addition to investigating children and young patients [45]. Therefore, in this work, we focus on using Xray images while keeping $\mathrm{CT}$ images as future work.

\section{CNN-BASED PROPOSED MODEL}

\subsection{Convolutional neural networks (CNNs)}

Artificial intelligence (AI) can contribute to the fight against COVID-19 in various ways such as immediate alarms and warnings, examination and prediction, following and forecast, treatments, and cures, data dashboards, and social control. Machine learning (ML) uses algorithms to analyze big-data and learn from it [46]. Then, it will be able to make a prediction or a decision about new unseen data [47]. A wellknown technique to realize machine learning is deep learning (DL), which employs algorithms caused by the composition and functionality of the neurons of the human brain. Artificial neural networks (ANN) are DL models that are based on the structure of the brain's neural network, which is a lightweight design for data classification problems. For a given set of inputs, the activation function of a neuron specifies its result. The activation function is inspired by the activity of our brain, where several neurons fire, i.e., are operated, by several stimuli. ANNs are an expensive solution for image classification problems since 2D images are converted to $1 \mathrm{D}$ vectors. Thus, 1D vectors will have a large number of trainable parameters with unusual storage and processing requirement. On the other hand, CNN, i.e., ConvNets, are a specific type of ANN that use convolution operation instead of the common multiplication in one of their layers at least. Thus, in this work, we implement CNN in Python.

The fundamental construction block for $\mathrm{CNN}$ are filters, i.e., kernels, which are applied to deduce the related features from the input images utilizing the convolution process. CNNs include two main steps, where the first step is feature learning, which includes: i) convolution for features learning, ii) application of activation function to obtain non-linearity, and iii) pooling to decrease dimensionality and maintain spatial invariance. The second step is the classification, where the output of the feature extraction is fed into fully connected layers. Then, the output is expressed as the chance of an image pertaining to an appropriate class. Generally, the CNNs have various advantages clouding their ability to automatically learn the filters which are used for feature extraction from the inputs. Moreover, CNNs capture the spatial features from images, i.e., the organization of pixels and the correlation between them. Besides, an individual filter is employed across various parts on input images to compose a feature map, i.e., parameter sharing. Section 3.4 explains the architecture of the proposed CNN model, including feature extraction and classification. Moreover, Table 1 explains the parameters of the layers of the proposed/constructed model.

A well-known problem in ML-based models is overfitting and underfitting. Model overfitting is when the model has high prediction accuracy for the training data while it has low prediction accuracy for the testing data. Furthermore, underfitting is when the model is unable to properly predict the class of the data even for the data it was trained on. To solve model underfitting we could increase the complexity of the model or add more features to the input sample. Data augmentation is achieved by making a conscious modification to the training data, e.g., flip, rotate, crop, zoom, and vary the color, in order to produce new data and reduce model overfitting. Thus, we ensured that our model is free from over- \& under-fitting. Figure 3 shows the main phases of our CNN-based proposed design. It includes six main steps, which are data preprocessing, importing the necessary modules, data partitioning, building the model by iteratively adding layers, fitting the model, and building a Flask webpage as a graphical user interface (GUI).

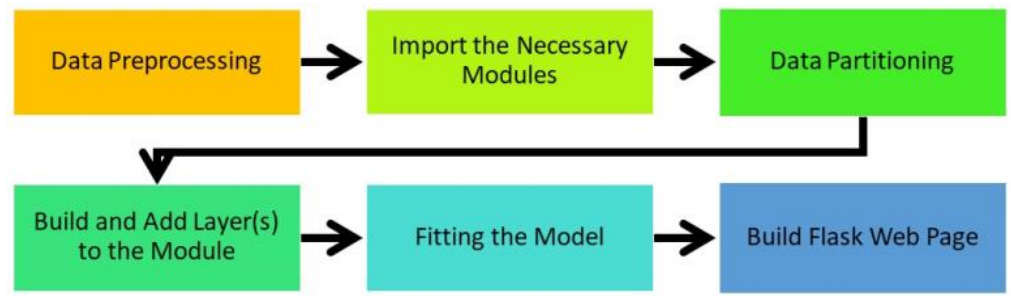

Figure 3. The main phases of the CNN-based proposed design

\subsection{Data Set of $X$-ray images}


In this work, we handled chest X-ray images of three classes, i.e., normal, viral pneumonia, and COVID-19 infected where Figure 4 depicts a sample set of the used images. For the collected dataset which covers three classes, we collect $1345 \mathrm{X}$-ray images of the normal class, $1342 \mathrm{X}$-ray images of the pneumonia class, and 217 images of the COVID-19 class. Thus, the complete number of images of the dataset is 2905 . This data has been obtained from the Kaggle repository [48] and used by previous works, e.g., [32]-[40]. In the exploratory analysis we divide the data into a training set of 2324 images, i.e., $80 \%$ of the data, and a validation set of 581 images, i.e., 20\% of the data such data partitioning is used by most of ML models and various related works. For parameter tuning, we used 464 images while performed final model testing by the remaining 117 images.

Regarding data preprocessing, we exported the X-ray images, with their APIs, from the Kaggle repository [48]. Then, we cleaned the exported data. It is well-known that DL methods require a large dataset of images to obtain a reliable result, which is not always available due to privacy issues, cost, and data generation time. Thus, we augmented the data through performing data transformation, e.g., rotating and scaling, to extend the size of the training data. Moreover, data enlargement solves the problem of model overfitting and improves the achieved accuracy.

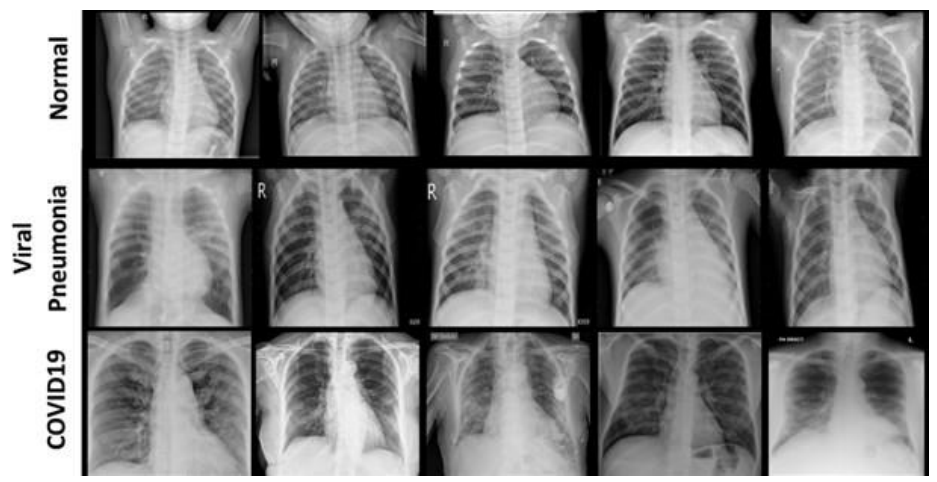

Figure 4. A sample of data set of X-ray images

\subsection{Import the required modules}

We used Keras, which is a deep learning Python library. It offers simple and compatible application programming interface (API) with easily explained error messages. Thus, the required user actions are minimal. If needed, comprehensive documentation and guidance are also available. Keras enables a fast transition from the idea to the result. We used Adam as an optimization algorithm and Relu as an activation function. Moreover, we used various libraries as explained next.

With images, efficient computing requires performing operations on data as vectors and matrices. Thus, linear algebra is required where the scientific computing library, i.e., NumPy, is utilized. Communicating with the operating system is achieved by various functions implemented by the OS module in Python. For data science and analytics, we use the Pandas module which runs on top of the NumPy library. The sequential module allows creating a deep learning model by adding layers to it. However, some architectures are not linear stacks, e.g., a Siamese NN, which concurrently works on two different input vectors. However, in this work, we use the sequential model. Flatten library converts multi-dimension matrices to vectors.

\subsection{Developing deep learning-based model}

For the implementation of the target design, we considered various features, i.e., design specifications, that are related to the neural network and the associated web page. The main three features are: i) time complexity where an efficient algorithm will have a short execution time, ii) performance where a high prediction accuracy is obtained by changing the number of layers of the NN, the number of the nodes per layer, and the type of the activation function, and iii) novelty where a new design is implemented without using previous designs.

We obtained the data from the Kaggle repository, cleaned it, and performed data augmentation. Then, this data is applied to train the suggested model. For more useful investigation, we implemented various models with different settings. Then, we examine their performance to determine the accuracy. We plan to reduce the loss function with succeeding epochs, where one Epoch is when a complete dataset is 
moved forward and backward within the neural network once. Therefore, we adopted Adam optimizer with a learning rate $(\mathrm{LR})=0.001$ for model training. We have employed the categorical cross-entropy loss to train our model. In the proposed models, we used the default activation function, i.e., the rectified linear unit (ReLU), which is shown in Figure 5 where $y=\operatorname{Max}(0, x)$. Figure 6 shows the structure of the proposed model where more implementation details are given in Table 1.

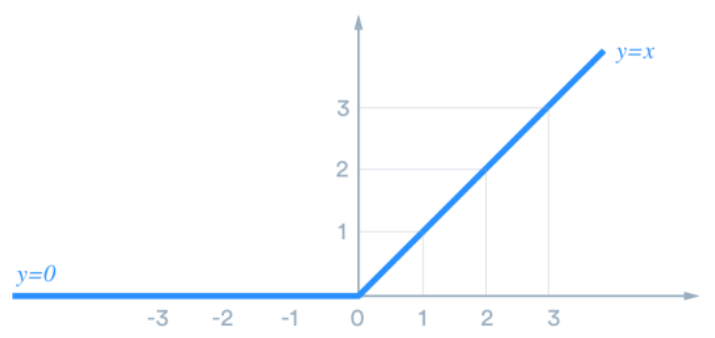

Figure 5. Activation functions: ReLU

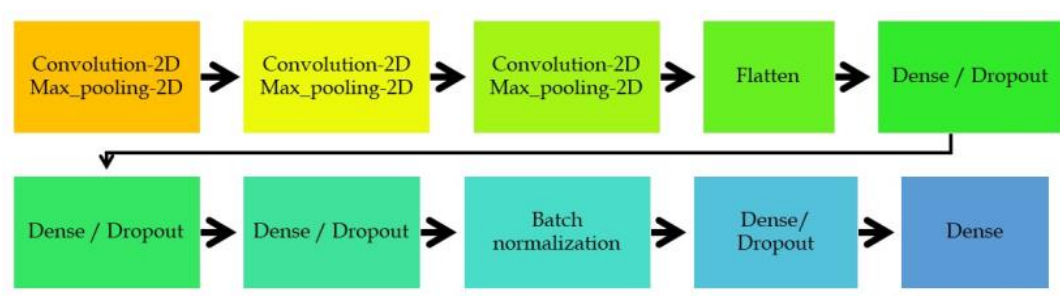

Figure 6. The architecture of the proposed CNN Model

Table 1. Parameters of the layers of the proposed model.

\begin{tabular}{cccc}
\hline Number of Layers & Layer (Type) & Output Shape & Number of Trainable Parameters \\
\hline 1 & Conv-2D & $(222,222,32)$ & 896 \\
2 & Max pool-2D & $(111,11,32)$ & 0 \\
3 & Conv-2D & $(109,109,64$ & 18496 \\
4 & Max pool-2D & $(36,36,64)$ & 0 \\
5 & Conv-2D & $(34,34,64)$ & 36928 \\
6 & Max pool-2D & $(17,17,64)$ & 0 \\
7 & Flatten & $(18496)$ & 0 \\
8 & Dense & $(512)$ & 9470464 \\
9 & Dropout & $(512)$ & 0 \\
10 & Dense & $(256)$ & 131328 \\
11 & Dropout & $(256)$ & 0 \\
12 & Dense & $(128)$ & 32896 \\
13 & Dropout & $(128)$ & 0 \\
14 & Batch Normalization & $(128)$ & 512 \\
15 & Dense & $(64)$ & 8256 \\
16 & Dropout & $(64)$ & 0 \\
17 & Dense & $(3)$ & 195 \\
\hline
\end{tabular}

Padding is a technique that allows us to keep the size of the filtered image the same as the original image. However, we did not use padding in any convolutional layer $(\mathrm{P}=0)$. This is why for an input image of size $n \times n$, we obtain an output image of size $n-2 \times n-2$. Application of the filter to the input image requires moving the filter from left to right and from top to bottom. The amount of this movement is defined as stride. The default value of the stride is $(1,1)$ for the horizontal and vertical movement, where such value works well for most of the cases. Therefore, we applied the default value of the filters in all convolutional layers in this work. When performing a stride convolution for an input image of size $n \times n$, padding is (P), the stride is (S), and the filter size is F×F. Then the size of the output is defined as in (1).

$$
\text { Output size }=\left(\frac{n+2 P-F}{s}+1\right)\left(\frac{n+2 P-F}{s}+1\right)
$$

As shown in Figure 6, we used convolution and maximum pooling thrice, where the used X-ray images are of size $224 \times 224$ pixels. The size of the filter for the three convolutional layers is $3 \times 3$ while $S=1$. 
For the first layer, the convolution result is an images of size $222 \times 222$. Then, we applied the max pool function with $\mathrm{S}=2$ and $\mathrm{F}=2$, utilizing 32 filters. The result of maximum pooling is images of size of $111 \times 111$. Regarding the second layer, the max pool function has $\mathrm{S}=3$ and $\mathrm{F}=3$, utilizing 64 filters. On the other hand, the third layer has $\mathrm{S}=2$ and $\mathrm{F}=2$, which is similar to the first layer. The output shape of the 3 layers is shown in Table 1, which are directly obtained according to (1).

The result of applying 2D convolution and max-pooling has the size of $(17,17,64)$. Then, Flatten which converts multi-dimension matrices to vector, is applied to convert the data from $17 \times 17 \times 64$ to 18496 . The dense, i.e., fully connected layer, which is a linear operation on the layer's input vector, is used 5 times (layer $8,10,12,15$ and 17). The input vector for the first dense layer is 512 which is reduced by 2 to reach 64 for the 4th dense layer, while the 5th dense layer has an output vector of 3 which represents one class of the $\mathrm{X}$-ray possible types. Dropout is a well-known regularization procedure, which aims to decrease the complexity of the model with the aim to stop overfitting. Thus, we applied dropout 4 times directly after applying dense operation. Batch normalization technique is designed to standardize the inputs to a layer in CNN. Thus, it significantly expediting the training process with improved performance. In our model, we applied batch normalization once after the third pair of dense-dropout.

\section{RESULTS AND DISCUSSION}

Figure 7 shows the training loss and validation loss for the 30 epochs of model training. We notice that the training loss starts with the maximum of 1.83 for the first epoch and reaches 0.073 for the last epoch. Regarding the validation loss, it starts by a value of 0.74 and reaches a maximum of 1.46 at epoch 11 . Then, the loss starts decreasing until it reaches 0.155 at the last epoch.

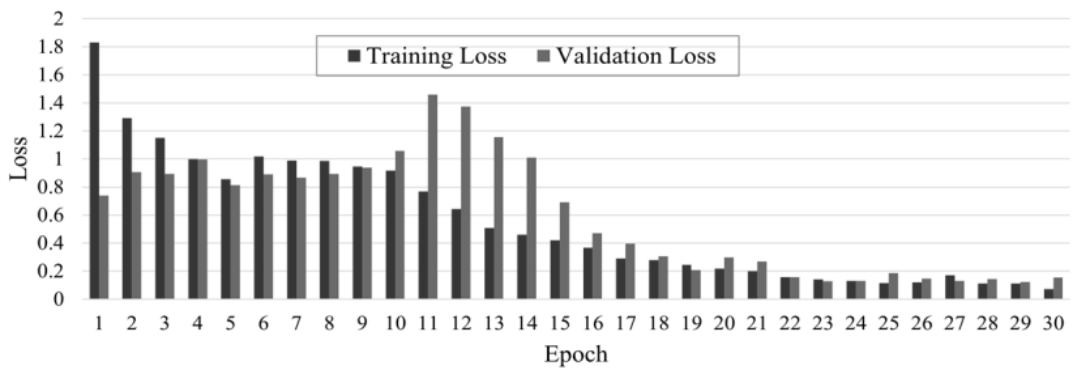

Figure 7. The training and validation loss of the model with successive epochs

Figure 8 shows the training and validation accuracy for the 30 epochs of model training. Regarding the accuracy of the training model, it starts by $32.1 \%$ and reaches the maximum of $97.55 \%$ at the last epoch. The last step was building a flask web page, where flask is a web framework written in Python. It does not require specific tools or libraries, does not include an abstraction method for the database, and it is a reliable framework for web users. We evaluated the proposed model based on accuracy as given in (2). The obtained confusion matrix, for evaluating the accuracy of the model on the testing data, is shown in Figure 9. The achievable accuracy is $97.44 \%$ for testing while the accuracy for the training was $97.55 \%$ as shown in Figure 8 .

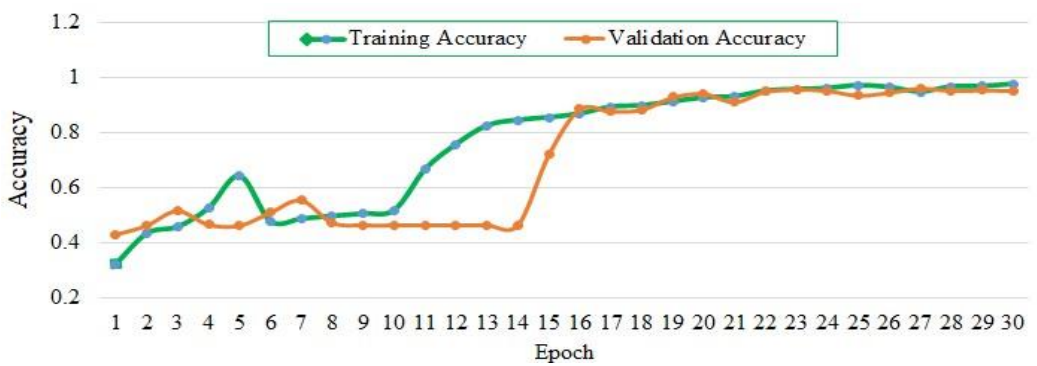

Figure 8 . The training and validation accuracy of the model with successive epochs 
The model is called though an interface using flask page as shown in Figure 10. Accordingly, Figures 10(a), 10(b), and 10(c) show the results provided by the model for given images, where these images are classified as normal, COVID-19, and viral pneumonia, respectively. In section 2. we explained various works which are closely related to this work. Table 2 shows a tabular comparison of the proposed model with various related work, i.e., based on chest X-ray and CT images. Moreover, the table shows the number of used images, the type of the images, the used method, and the final accuracy of the model.

\begin{tabular}{|c|c|c|c|c|}
\cline { 3 - 5 } \multicolumn{2}{c|}{} & \multicolumn{3}{c|}{ Predicted Class } \\
\cline { 3 - 5 } \multicolumn{2}{c|}{} & COVID-19 & Normal & $\begin{array}{c}\text { Viral } \\
\text { Pneumonia }\end{array}$ \\
\hline \multirow{3}{*}{$\begin{array}{c}\text { Actual } \\
\text { Class }\end{array}$} & COVID-19 & 10 & 0 & 0 \\
\cline { 2 - 5 } & Normal & 1 & 51 & 1 \\
\cline { 2 - 5 } & $\begin{array}{c}\text { Viral } \\
\text { Pneumonia }\end{array}$ & 1 & 0 & 53 \\
\hline
\end{tabular}

Figure 9. The confusion matrix of the testing data

$$
\text { Accuracy }=\frac{\text { True Positive }+ \text { True Negative }}{\text { True Positive }+ \text { True Negative }+ \text { False Positive }+ \text { False Negative }}
$$

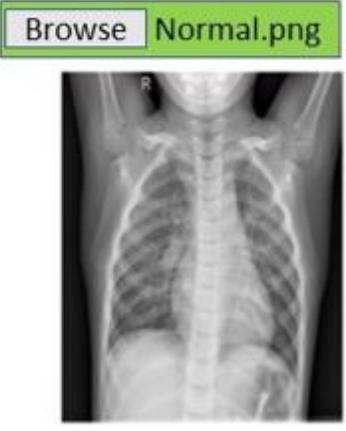

(a)

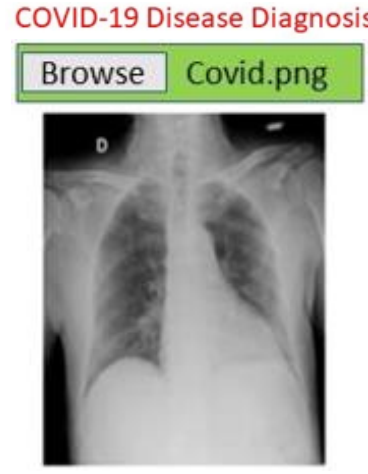

(b)

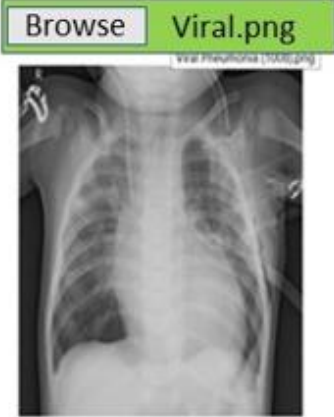

(c)

Figure 10. The GUI of the flask page for COVID-19 detection (a) result: normal, (b) result: COVID-19, and (c) result: viral pneumonia

Table 2. Comparison between related DL models for covid-19 identification

\begin{tabular}{ccccc}
\hline Work & Number of Cases & Type of Images & Method & Accuracy (\%) \\
\hline$[33]$ & 50 & X-ray & COVIDX-Net & 90 \\
{$[35]$} & 170 & X-ray & CNN & 94.1 \\
{$[36]$} & 13645 & X-ray & COVID-Net & 92.4 \\
{$[37]$} & 1427 & X-ray & VGG-19 & 96.78 \\
{$[38]$} & 50 & X-ray & ResNet50+SVM & 95.33 \\
{$[39]$} & 100 & X-ray & InceptionV3 & 95.4 \\
{$[39]$} & 100 & X-ray & ResNet50 & 96.1 \\
{$[40]$} & 4376 & X-ray & ResNet-101 & 71.9 \\
{$[41]$} & 1485 & CT & DRE-Net & 86 \\
{$[42]$} & 453 & CT & M-Inception & 82.9 \\
{$[43]$} & 542 & CT & UNet+3D Deep Network & 90.8 \\
{$[44]$} & 618 & CT & ResNet + Location Attention & 86.7 \\
Proposed & 2905 & X-ray & CNN & 97.44 \\
\hline
\end{tabular}

Various authors used the dataset [48] for Covid detection, where they utilized standard CNN architectures, e.g., ResNet. Because of its compelling results, ResNet quickly became one of the most popular architectures in various computer vision tasks. Although ResNet has proven powerful in many applications, one major drawback is that a deeper network usually requires weeks for training, making it practically infeasible in real-world applications. ResNet-50 is a CNN that is 50 layers deep, while ResNet$101 \mathrm{CNN}$ is 101 layers deep. We evaluated the accuracy of our proposed architecture using datasets of 
previous related works, i.e., [33]-[40]. We obtained a competitive accuracy. The use of mobile phones to scan X-ray images for the detection of COVID-19 is considered as an extension of this work.

\section{CONCLUSION}

The latest world pandemic of COVID-19 is progressing daily without a $100 \%$ efficient vaccine that has no side effect. Therefore, it is important to quickly identify the infected people to decrease the extent of the COVID-19 by prevention procedures and providing the required health care for the infected ones. Most countries, especially those that have limited resources, are unable to provide the required fast, cheap, and accurate tests to detect the infected cases. Therefore, in this paper, based on a limited set of chest X-ray scans, we investigated various CNN models to detect COVID-19 cases. Our proposed design is completely automated including automatic feature extraction. Moreover, the build models can classify the X-ray into infected, normal, or pneumonia, i.e., three-class classification, with a test accuracy of $97.44 \%$ and training accuracy of $97.55 \%$. We tested the proposed model/framework on various data sets which are used by previous works. The testing accuracy was indistinguishable with the accuracy of testing the model based on the training data. Our CNNbased model is usable by radiologists to aid in confirming their initial screening of patients where a negligible accuracy loss is accepted. Thus, this approach is applicable for automatic disease diagnosis e.g., remote places affected by COVID-19. Moreover, we could use it to diagnose other chestrelated diseases. To verify the high accuracy obtained, we tested the models based on the newly introduced public data and obtained almost the same accuracy. The main limitation of the work is using a limited amount of labelled X-ray images for COVID19. Thus, future work will make the model more accurate by using more images. Moreover, the use of magnetic resonance imaging (MRI) and computed tomography (CT) images could be investigated in addition to X-ray images. Finally, COVID-19 belongs to the same family of severe acute respiratory syndrome coronavirus (SARS-CoV) and Middle East Respiratory Syndrome-related Coronavirus (MERS-CoV). Thus, it is possible to detect SARS-CoV and MERS-CoV utilizing chest $\mathrm{X}$-ray and the proposed model/framework.

\section{REFERENCES}

[1] S. Bagcchi, "The world's largest COVID-19 vaccination campaign," The Lancet Infectious Diseases, vol. 21, no. 3, p. 323, Mar. 2021, doi: 10.1016/S1473-3099(21)00081-5.

[2] "How effective are the covid-19 vaccines?" 2021, Accessed: Mar. 25, $2021 . \quad$ [Online]. Available: https://www.statista.com/chart/23510/estimatedeffectiveness-of-covid-19-vaccine-candidates/.

[3] S. A. Gómez-Ochoa et al., "COVID-19 in health-care workers: a living systematic review and meta-analysis of prevalence, risk factors, clinical characteristics, and outcomes," American Journal of Epidemiology, vol. 190, no. 1, pp. 161-175, Jan. 2021, doi: 10.1093/aje/kwaa191.

[4] C. Vianello et al., "A perspective on early detection systems models for COVID-19 spreading," Biochemical and Biophysical Research Communications, vol. 538, pp. 244-252, Jan. 2021, doi: 10.1016/j.bbrc.2020.12.010.

[5] B. Aksoy and O. K. M. Salman, "Detection of COVID-19 disease in chest X-Ray Images with capsul networks: application with cloud computing," Journal of Experimental \& Theoretical Artificial Intelligence, vol. 33, no. 3, pp. 527-541, May 2021, doi: 10.1080/0952813X.2021.1908431.

[6] C. D. L. Goulart et al., "Lifestyle and rehabilitation during the COVID-19 pandemic: guidance for health professionals and support for exercise and rehabilitation programs," Expert Review of Anti-infective Therapy, vol. 19, no. 11, pp. 1385-1396, Nov. 2021, doi: 10.1080/14787210.2021.1917994.

[7] C. Basile et al., "Recommendations for the prevention, mitigation and containment of the emerging SARS-CoV-2 (COVID-19) pandemic in haemodialysis centres," Nephrology Dialysis Transplantation, vol. 35, no. 5, pp. 737-741, May 2020, doi: 10.1093/ndt/gfaa069.

[8] L. Yan et al., "Prediction of criticality in patients with severe Covid-19 infection using three clinical features: a machine learningbased prognostic model with clinical data in Wuhan," medRxiv, 2020, doi: 10.1101/2020.02.27.20028027.

[9] Y. M. Arabi et al., "How the COVID-19 pandemic will change the future of critical care," Intensive Care Medicine, vol. 47, no. 3, pp. 282-291, Mar. 2021, doi: 10.1007/s00134-021-06352-y.

[10] V. Kumar Singh, M. Abdel-Nasser, N. Pandey, and D. Puig, "LungINFseg: segmenting COVID-19 infected regions in lung CT Images based on a receptive-field-aware deep learning framework," Diagnostics, vol. 11, no. 2, p. 158, Jan. 2021, doi: 10.3390/diagnostics11020158.

[11] S. Bhattacharya et al., "Deep learning and medical image processing for coronavirus (COVID-19) pandemic: A survey," Sustainable Cities and Society, vol. 65, p. 102589, Feb. 2021, doi: 10.1016/j.scs.2020.102589.

[12] T. Hoshina et al., "Intensive diagnostic management of coronavirus disease 2019 (COVID-19) in academic settings in Japan: challenge and future," Inflammation and Regeneration, vol. 40, no. 1, pp. 38-47, Dec. 2020, doi: 10.1186/s41232-020-00147-2.

[13] L. Craxì, A. Casuccio, E. Amodio, and V. Restivo, "Who should get COVID-19 vaccine first? a survey to evaluate hospital workers' opinion," Vaccines, vol. 9, no. 3, p. 189, Feb. 2021, doi: 10.3390/vaccines9030189.

[14] M. Masadeh, O. Hasan, and S. Tahar, "Approximation-conscious IC testin," in 2018 30th International Conference on Microelectronics (ICM), Dec. 2018, pp. 56-59, doi: 10.1109/ICM.2018.8704099.

[15] T. Singhal, "A review of coronavirus disease-2019 (COVID-19)," The Indian Journal of Pediatrics, vol. 87, no. 4, pp. 281-286, Apr. 2020, doi: 10.1007/s12098-020-03263-6.

[16] D. R. Seshadri et al., "Wearable sensors for COVID-19: a call to action to harness our digital infrastructure for remote patient monitoring and virtual assessments," Frontiers in Digital Health, vol. 2, pp. 1-8, Jun. 2020, doi: 10.3389/fdgth.2020.00008. 
[17] M. Masadeh, O. Hasan, and S. Tahar, "Machine learning-based self-compensating approximate computing," pp. 1-6, Jan. 2020, [Online]. Available: http://arxiv.org/abs/2001.03783.

[18] M. Masadeh, O. Hasan, and S. Tahar, "Machine-Learning-based self-tunable design of approximate computing," IEEE Transactions on Very Large Scale Integration (VLSI) Systems, vol. 29, no. 4, pp. 800-813, Apr. 2021, doi: 10.1109/TVLSI.2021.3056243.

[19] O. M. AlShorman and A. M. Alshorman, "Frontal lobe and long-term memory retrieval analysis during pre-learning stress using EEG signals," Bulletin of Electrical Engineering and Informatics, vol. 9, no. 1, pp. 141-145, Feb. 2020, doi: 10.11591/eei.v9i1.1335.

[20] O. AlShorman et al., "Frontal lobe real-time eeg analysis using machine learning techniques for mental stress detection," Journal of Integrative Neuroscience, pp. 1-11, 2021.

[21] A. Yahyaoui, A. Jamil, J. Rasheed, and M. Yesiltepe, "A decision support system for diabetes prediction using machine learning and deep learning techniques," in 2019 1st International Informatics and Software Engineering Conference (UBMYK), Nov. 2019, pp. 1-4, doi: 10.1109/UBMYK48245.2019.8965556.

[22] M. Gurbina, M. Lascu, and D. Lascu, "Tumor detection and classification of MRI Brain image using different wavelet transforms and support vector machines," in 2019 42nd International Conference on Telecommunications and Signal Processing (TSP), Jul. 2019, pp. 505-508, doi: 10.1109/TSP.2019.8769040.

[23] L. Nanni, S. Ghidoni, and S. Brahnam, "Handcrafted vs. non-handcrafted features for computer vision classification," Pattern Recognition, vol. 71, pp. 158-172, Nov. 2017, doi: 10.1016/j.patcog.2017.05.025.

[24] B. Guragai, O. AlShorman, M. Masadeh, and M. B. Bin Heyat, "A survey on deep learning classification algorithms for motor imagery," in 2020 32nd International Conference on Microelectronics (ICM), Dec. 2020, pp. 1-4, doi: 10.1109/ICM50269.2020.9331503.

[25] K. Mahmoud et al., "Prediction of the effects of environmental factors towards COVID-19 outbreak using AI-based models," IAES International Journal of Artificial Intelligence (IJ-AI), vol. 10, no. 1, p. 35, Mar. 2021, doi: 10.11591/ijai.v10.i1.pp35-42.

[26] H. Trung Huynh and V. Nguyen Nhat Anh, "A deep learning method for lung segmentation on large size chest X-ray image," in 2019 IEEE-RIVF International Conference on Computing and Communication Technologies (RIVF), Mar. 2019, pp. 1-5, doi: 10.1109/RIVF.2019.8713648.

[27] D. Bardou, K. Zhang, and S. M. Ahmad, "Classification of breast cancer based on histology images using convolutional neural networks," IEEE Access, vol. 6, pp. 24680-24693, 2018, doi: 10.1109/ACCESS.2018.2831280.

[28] B. Acs, M. Rantalainen, and J. Hartman, "Artificial intelligence as the next step towards precision pathology," Journal of Internal Medicine, vol. 288, no. 1, pp. 62-81, Jul. 2020, doi: 10.1111/joim.13030.

[29] H. Salehinejad, E. Colak, T. Dowdell, J. Barfett, and S. Valaee, "Synthesizing chest X-Ray pathology for training deep convolutional neural networks," IEEE Transactions on Medical Imaging, vol. 38, no. 5, pp. 1197-1206, May 2019, doi: 10.1109/TMI.2018.2881415

[30] C. Shorten and T. M. Khoshgoftaar, "A survey on image data augmentation for deep learning," Journal of Big Data, vol. 6, no. 1, p. 60 , Dec. 2019, doi: 10.1186/s40537-019-0197-0.

[31] H.-C. Shin et al., "Deep convolutional neural networks for computer-aided detection: cnn architectures, dataset characteristics and transfer learning," IEEE Transactions on Medical Imaging, vol. 35, no. 5, pp. 1285-1298, May 2016, doi: 10.1109/TMI.2016.2528162.

[32] L. N. Mahdy, K. A. Ezzat, H. A. E. Haytham H. Elmousalami, and E. Hassanien, "Automatic X-ray COVID-19 lung image classification system based on multi-level thresholding and support vector machine," medRxiv, 2020.

[33] E. E.-D. Hemdan, M. A. Shouman, and M. E. Karar, "COVIDX-Net: A framework of deep learning classifiers to diagnose COVID-19 in X-Ray images," Mar. 2020, [Online]. Available: http://arxiv.org/abs/2003.11055.

[34] M. Ilyas, H. Rehman, and A. Nait-ali, "Detection of Covid-19 from chest X-ray images using artificial intelligence: an early review," Apr. 2020, [Online]. Available: http://arxiv.org/abs/2004.05436.

[35] H. S. Maghdid, A. T. Asaad, K. Z. Ghafoor, A. S. Sadiq, and M. K. Khan, "Diagnosing COVID-19 pneumonia from X-Ray and CT images using deep learning and transfer learning algorithms," Mar. 2020, [Online]. Available: http://arxiv.org/abs/2004.00038.

[36] L. Wang, Z. Q. Lin, and A. Wong, "COVID-Net: a tailored deep convolutional neural network design for detection of COVID-19 cases from chest X-ray images," Scientific Reports, vol. 10, no. 1, pp. 11961-19549, Dec. 2020, doi: 10.1038/s41598-020-76550Z.

[37] I. D. Apostolopoulos and T. A. Mpesiana, "Covid-19: automatic detection from X-ray images utilizing transfer learning with convolutional neural networks," Physical and Engineering Sciences in Medicine, vol. 43, no. 2, pp. 635-640, Jun. 2020, doi: 10.1007/s13246-020-00865-4.

[38] P. K. Sethy and S. K. Behera, "Detection of coronavirus disease (covid-19) based on deep features and support vector machine," Preprints, 2020, doi: 10.20944/preprints202003.0300.v1.

[39] A. Narin, C. Kaya, and Z. Pamuk, "Automatic detection of coronavirus disease (COVID-19) using X-ray images and deep convolutional neural networks," Pattern Analysis and Applications, vol. 24, no. 3, pp. 1207-1220, Aug. 2021, doi: 10.1007/s10044-021-00984-y.

[40] M. Z. Che Azemin, R. Hassan, M. I. Mohd Tamrin, and M. A. Md Ali, "COVID-19 deep learning prediction model using publicly available radiologist-adjudicated chest X-Ray images as training data: preliminary findings," International Journal of Biomedical Imaging, vol. 2020, pp. 1-7, Aug. 2020, doi: 10.1155/2020/8828855.

[41] S. Ying et al., "Deep learning enables accurate diagnosis of novel coronavirus (covid-19) with ct images," medRxiv, 2020, doi: 10.1101/2020.02.23.20026930.

[42] S. Wang et al., "A deep learning algorithm using CT images to screen for corona virus disease (COVID-19)," European Radiology, vol. 31, no. 8, pp. 6096-6104, Aug. 2021, doi: 10.1007/s00330-021-07715-1.

[43] C. Zheng et al., "Deep learning-based detection for covid-19 from chest ct using weak label," medRxiv, 2020, doi: 10.1101/2020.03.12.20027185.

[44] X. Xu et al., "A deep learning system to screen novel coronavirus disease 2019 pneumonia," Engineering, vol. 6, no. 10, pp. 1122-1129, Oct. 2020, doi: 10.1016/j.eng.2020.04.010.

[45] Y. A. Vasilev et al., "Chest MRI of patients with COVID-19," Magnetic Resonance Imaging, vol. 79, pp. 13-19, Jun. 2021, doi: 10.1016/j.mri.2021.03.005.

[46] S. R. Salkuti, "A survey of big data and machine learning," International Journal of Electrical and Computer Engineering (IJECE), vol. 10, no. 1, pp. 575-580, Feb. 2020, doi: 10.11591/ijece.v10i1.pp575-580

[47] F. T. Fernandes, T. A. de Oliveira, C. E. Teixeira, A. F. de M. Batista, G. Dalla Costa, and A. D. P. Chiavegatto Filho, "A multipurpose machine learning approach to predict COVID-19 negative prognosis in São Paulo, Brazil," Scientific Reports, vol. 
11, no. 1, pp. 3343-3350, Dec. 2021, doi: 10.1038/s41598-021-82885-y.

[48] "Novel Corona Virus 2019 Dataset." 2021, Accessed: Mar. 25, 2021. [Online]. Available: https://www.kaggle.com/sudalairajkumar/novel-corona-virus2019-dataset.

\section{BIOGRAPHIES OF AUTHORS}
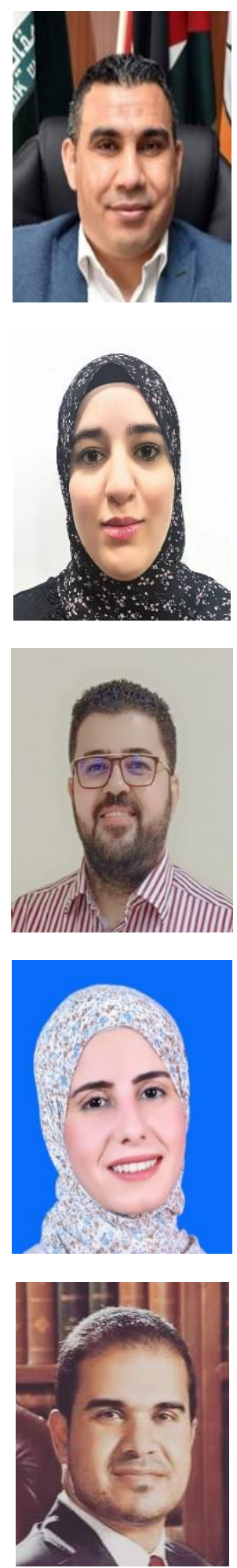

Mahmoud Masadeh (iD 8. SC P received a B.Sc. degree in Computer Engineering, in 2003, Jordan. He holds an M.Sc degree in Computer Engineering from the Delft University of Technology, The Netherlands, as well as an M.Sc, in Management Information Systems from Jordan. He served as a full-time Teaching Assistant (TA) from 2005 to 2011, and as a fulltime Instructor from 2013 to 2016, both at the Computer Engineering Department, Yarmouk University. In August 2020, he obtained his Ph.D. from Concordia University. Currently, he is an Assistant Professor at the Computer Engineering Department, Yarmouk University, Jordan. His research focuses on artificial intelligence and its application, smart health care, circuit design, approximate computing, and energy-efficient VLSI circuit design. Mahmoud is a member of IEEE, ACM and member of the Jordanian Engineering Association.

Ayah Masadeh (ID 8) SC P received a B.Sc. degree in Computer Engineering, in 2021, from Yarmouk University, Jordan. Currently, she is working as an independent reserchers. Her research focuses on machine learning, artificial intelligence, and hardware design.

Omar Alshorman (D) 8D SC P was born in Irbid, Jordan in 1986. He received a B.S. degree in computer engineering from Al-Hussien Bin Talal University - Jordan in 2009, and M.S. from Yarmouk University- Jordan in 2012. In 2014, he joined an electrical engineering department, faculty of engineering, Najran University, Najran, Saudi Arabia as a lecturer. Currently, he is working as a project manager at AlShrouk trading company, Najran University, Najran, Saudi Arabia. His research interests are signal and image processing, healthcare informatics, the internet of things, artificial intelligence, and condition monitoring.

Falak H Khasawneh (iD) 8 SC $\mathrm{P}$ received a B.S. degree in medical laboratory sciences from Jordan University of Science and Technology, Jordan in 2014, and M.Sc. in clinical biochemistry/ Medical laboratory Sciences from Jordan University of Science and Technology, Jordan 2017. She served part time lecturer from 2017-2021 at medical laboratory sciences department at Jordan University of Science and Technology, and as full-time lecturer at Zarqa university college, Balqa applied university from 2021-now. Her research fields include many areas of laboratory sciences such as: male fertility, drug effects, infectious diseases, and healthcare.

Mahmud Ali Masadeh (D) 8 SC P received a B.S. degree in computer science from Irbid National University, Jordan in 2009, and M.Sc in Information and Communication Technology in Education from Al Albayt University, Jordan in 2018. Currently, he is working at Ministry of Education. His research interests are artificial intelligence and its application, Eeducation, and E-health. 